\section{Labile plasma iron levels predict survival in patients with lower-risk myelodysplastic syndromes}

Louise de Swart, ${ }^{1}$ Chloé Reiniers, ${ }^{2}$ Timothy Bagguley, ${ }^{3}$ Corine van Marrewijk, ${ }^{1}$ David Bowen, ${ }^{4}$ Eva Hellström-Lindberg, ${ }^{5}$ Aurelia Tatic, ${ }^{6}$ Argiris Symeonidis, ${ }^{7}$ Gerwin Huls, ${ }^{2}$ Jaroslav Cermak, ${ }^{8}$ Arjan A. van de Loosdrecht, ${ }^{9}$

Hege Garelius, ${ }^{10}$ Dominic Culligan, ${ }^{11}$ Mac Macheta, ${ }^{12}$ Michail Spanoudakis,${ }^{13}$ Panagiotis Panagiotidis, ${ }^{14}$ Marta Krejci, ${ }^{15}$ Nicole Blijlevens, ${ }^{1}$

Saskia Langemeijer, ${ }^{1}$ Jackie Droste,${ }^{1}$ Dorine W. Swinkels, ${ }^{16}$ Alex Smith ${ }^{2}$ and Theo de Witte ${ }^{17}$ on behalf of the EUMDS Steering Committee

${ }^{1}$ Department of Hematology, Radboud university medical center, Nijmegen, the Netherlands; 'Department of Hematology, University Medical Centre, Groningen, the Netherlands; ${ }^{3}$ Epidemiology and Cancer Statistics Group, University of York, UK; ${ }^{4}$ St. James's Institute of Oncology, Leeds Teaching Hospitals, UK; ${ }^{5}$ Department of Medicine, Division of Hematology, Karolinska Institutet, Stockholm, Sweden; ${ }^{6}$ Center of

Hematology and Bone Marrow Transplantation, Fundeni Clinical Institute, Bucharest, Romania; 'Department of Medicine, Division of Hematology, University of Patras Medical School, Greece; ${ }^{8}$ Department of Clinical Hematology, Institute of Hematology \& Blood Transfusion, Prague, Czech Republic; ${ }^{9}$ Department of Hematology - Cancer Center Amsterdam VU University Medical Center, The Netherlands; ${ }^{10}$ Department of Medicine, Section of Hematology and Coagulation, Sahlgrenska University Hospital, Göteborg, Sweden; ${ }^{11}$ Department of Haematology, Aberdeen Royal Infirmary, UK; ${ }^{12}$ Department of Haematology, Blackpool Victoria Hospital, Lancashire, UK;

${ }^{13}$ Department of Haematology, Airedale NHS trust, UK; ${ }^{14}$ Department of Hematology, Laikon General Hospital, National and Kapodistrian University of Athens, Greece; ${ }^{15}$ Department of Internal Medicine, Hematology and Oncology, University Hospital Brno and Masaryk University, Czech Republic; ${ }^{16}$ Department of Laboratory Medicine, Hepcidinanalysis.com, and Radboudumc expertise center for iron disorders, Radboud university medical center, Nijmegen, the Netherlands and ${ }^{17}$ Nijmegen Center for Molecular Life Sciences, Department of Tumor Immunology, Radboud university medical center, the Netherlands

\section{ABSTRACT}

$\mathrm{R}$ ed blood cell transfusions remain one of the cornerstones in supportive care of lower-risk patients with myelodysplastic syndromes. We hypothesized that patients develop oxidant-mediated tissue injury through the formation of toxic iron species, caused either by red blood cell transfusions or by ineffective erythropoiesis. We analyzed serum samples from 100 lower-risk patients with myelodysplastic syndromes at six-month intervals for transferrin saturation, hepcidin-25, growth differentiation factor 15 , soluble transferrin receptor, non-transferrin bound iron and labile plasma iron in order to evaluate temporal changes in iron metabolism and the presence of potentially toxic iron species and their impact on survival. Hepcidin levels were low in 34 patients with ringed sideroblasts compared to 66 patients without. Increases of hepcidin and non-transferrin bound iron levels were visible early in follow-up of all transfusion-dependent patient groups. Hepcidin levels significantly decreased over time in transfusion-independent patients with ringed sideroblasts. Increased soluble transferrin receptor levels in transfusion-independent patients with ringed sideroblasts confirmed the presence of ineffective erythropoiesis and suppression of hepcidin production in these patients. Detectable labile plasma iron levels in combination with high transferrin saturation levels occurred almost exclusively in patients with ringed sideroblasts and all transfusiondependent patient groups. Detectable labile plasma iron levels in transfusion-dependent patients without ringed sideroblasts were associated with decreased survival. In conclusion, toxic iron species occurred in all transfusion-dependent patients and in transfusion-independent patients with ringed sideroblasts. Labile plasma iron appeared to be a clinically relevant measure for potential iron toxicity and a prognostic factor for survival in transfusion-dependent patients. clinicaltrials.gov Identifier: 00600860 .

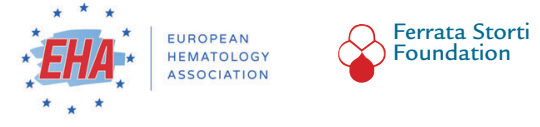

Haematologica 2018

Volume 103(1):69-79

\section{Correspondence:}

theo.dewitte@radboudumc.nl

Received: May 26, 2017.

Accepted: October 27, 2017.

Pre-published: November 9, 2017.

doi:10.3324/haematol.2017.171884

Check the online version for the most updated information on this article, online supplements, and information on authorship \& disclosures: www.haematologica.org/content/103/1/69

\section{(C)2018 Ferrata Storti Foundation}

Material published in Haematologica is covered by copyright. All rights are reserved to the Ferrata Storti Foundation. Use of published material is allowed under the following terms and conditions:

https://creativecommons.org/licenses/by-nc/4.0/legalcode. Copies of published material are allowed for personal or internal use. Sharing published material for non-commercial purposes is subject to the following conditions:

https://creativecommons. org//icenses/by-nc/4.0/legalcode, sect. 3. Reproducing and sharing published material for commercial purposes is not allowed without permission in writing from the publisher. 


\section{Introduction}

Myelodysplastic syndromes (MDS) are a heterogeneous group of acquired clonal hematopoietic stem cell disorders that are characterized by abnormal differentiation and maturation of hematopoietic cells, bone marrow failure and genetic instability with an enhanced risk of progression to acute myeloid leukemia. ${ }^{1}$ The European MDS (EUMDS) registry is a prospective, observational registry which was established in 2007 in order to collect data on low and intermediate-1-risk MDS patients, who represent the lower-risk MDS population, comprising approximately seventy percent of the overall MDS population. ${ }^{2,3}$ The majority of lower-risk MDS patients (51\% in the EUMDS Registry) ${ }^{3}$ become transfusion-dependent, usually early after diagnosis. With an expected median survival of 2.4 to 11.8 years, these patients are prone to long-term accumulation of iron due to red blood cell (RBC) transfusions. ${ }^{4.8}$ Iron overload may also occur in MDS patients who do not receive $\mathrm{RBC}$ transfusions, due to the stimulation of intestinal iron absorption, mediated through the suppression of hepcidin production in patients with ineffective erythropoiesis. ${ }^{9}$ Patients with ringed sideroblasts (MDS-RS) are of special interest in this context, considering their pronounced ineffective erythropoiesis..$^{67,10,11}$

The toxic effects of iron overload in other iron loading diseases, such as hereditary hemochromatosis ${ }^{11}$ and the thalassemia syndromes ${ }^{12}$ are well known, but the consequences in MDS remain to be elucidated. MDS patients are generally older than patients with other iron loading disorders. ${ }^{13}$ Their exposure may not be long enough to develop classical tissue damage due to iron overload, but they may suffer from oxidative stress caused by toxic iron molecules. Moreover, iron toxicity might be restricted to specific subgroups of MDS patients; those receiving RBC transfusions and a subgroup of patients with MDS-RS and increased ineffective erythropoiesis. ${ }^{5,13}$
A greater insight into the pathophysiology of iron metabolism in MDS might be obtained through an optimized diagnostic work-up and monitoring by specific iron metabolism markers, including hepcidin, growth differentiation factor 15 (GDF15), soluble transferrin receptor (sTFR), and the recently introduced serum toxic iron species, namely non-transferrin bound iron (NTBI) and labile plasma iron (LPI). ${ }^{14-18}$ The most important regulator of systemic iron metabolism is hepcidin, a 25-aminoacid peptide hormone, produced predominantly by the hepatocytes. Hepcidin triggers internalization and lysosomal degradation of ferroportin, a membrane bound cellular iron exporter present on macrophages and the basolateral site of enterocytes that releases iron into the circulation. ${ }^{19,20}$ Hepcidin is suppressed in hypoxia and with increased erythropoietic iron demand and is upregulated in case of inflammation and increased circulating iron levels and elevated body iron stores.

GDF15 is a protein produced by erythroid precursors and has been reported to be involved in the communication between bone marrow and liver in case of an increased erythroid demand, functioning as a suppressor of hepcidin synthesis, as shown for $\beta$-thalassemia. . $, 2,22$ However, its role in MDS is still a matter of debate due to conflicting results. ${ }^{5,11,22-25}$ Twisted gastrulation factor 1 (TWSG1) and erythroferrone (ERFE) are also reported to have a suppressive function in hepatic hepcidin production, however, validated human assays are not available. ${ }^{9}$ Of additional interest in iron homeostasis is sTFR. The serum concentration of sTFR is proportional to the quantity of the transferrin receptors 1 (TfR1) on cellular membranes, especially on erythroid precursors, and is a valuable parameter of erythroid mass and iron supplies. ${ }^{26,27}$ Among others, sTFR levels are elevated in case of high erythroid proliferation rates, especially in combination with adequate iron supply, ${ }^{27}$ as in diseases characterized by ineffective erythropoiesis, such as $\beta$-thalassemia syn-

Table 1. Frequency, median and quartiles of iron substudy parameters overall, by transfusion status and MDS subtype at first sample.

\begin{tabular}{|c|c|c|c|c|c|c|c|c|c|c|}
\hline & \multirow[b]{2}{*}{ N } & \multirow[b]{2}{*}{$\begin{array}{c}\text { Total } \\
\text { Median } \\
\text { (p10-p90) }\end{array}$} & \multicolumn{4}{|c|}{ Transfusion } & \multicolumn{4}{|c|}{ Ring Sideroblasts } \\
\hline & & & $\mathbf{N}$ & $\begin{array}{l}\text { Independent } \\
\text { Median } \\
\text { (p10-p90) }\end{array}$ & $\mathbf{N}$ & $\begin{array}{l}\text { Dependent } \\
\text { Median } \\
\text { (p10-p90) }\end{array}$ & N & $\begin{array}{c}\text { No } \\
\text { Median } \\
\text { (p10-p90) }\end{array}$ & N & $\begin{array}{c}\text { Yes } \\
\text { Median } \\
\text { (p10-p90) }\end{array}$ \\
\hline Hemoglobin ( $\mathrm{g} / \mathrm{dl})$ & 100 & $10.2(8.3-12.4)$ & 85 & $10.3(8.6-12.6)$ & 15 & $9.3(6.4-10.9)$ & 66 & $10.4(8.5-12.5)$ & 34 & $9.9(7.3-12.1)$ \\
\hline White blood cells $\left(10^{9} \mathrm{~L}\right)$ & 100 & $4.8(2.4-8.7)$ & 85 & $5.1(2.5-8.7)$ & 15 & $3.8(2.3-10.7)$ & 66 & $3.9(2.3-7.4)$ & 34 & $6.0(3.9-11.4)$ \\
\hline Platelets $\left(10^{9} / \mathrm{L}\right)$ & 99 & $212(94-475)$ & 84 & $218(97-475)$ & 15 & $158(87-463)$ & 66 & $168(89-341)$ & 33 & $316(169-501)$ \\
\hline Serum Iron $(\mu \mathrm{mol} / \mathrm{L})$ & 100 & $20(12-38)$ & 85 & $19(12-34)$ & 15 & $26.0(4.0-47.0)$ & 66 & $17(10-26)$ & 34 & $30(16-45)$ \\
\hline Ferritin $(\mu \mathrm{g} / \mathrm{L})$ & 100 & $287(48-982)$ & 85 & $264(49-692)$ & 15 & $634(20-1897)$ & 66 & $246(36-665)$ & 34 & $376(127-1242)$ \\
\hline Transferrin saturation (\%) & 100 & $36(19-87)$ & 85 & $35(19-81)$ & 15 & $52(13-93)$ & 66 & $31(17-61)$ & 34 & $59(25-93)$ \\
\hline Hepcidin $(\mathrm{nmol} / \mathrm{L})$ & 99 & $4.5(1.1-21.7)$ & 84 & $4.2(1.2-13.8)$ & 15 & $6.8(0.5-53.7)$ & 66 & $4.7(1.1-24.2)$ & 33 & $4.2(1.2-10.3)$ \\
\hline Soluble transferrin receptor (mg/L) & 100 & $1.3(0.7-2.8)$ & 85 & $1.3(0.8-2.8)$ & 15 & $0.9(0.6-3.0)$ & 66 & $1.2(0.7-2.7)$ & 34 & $1.5(0.8-3.1)$ \\
\hline C-reactive protein (mg/L) & 100 & $5.0(4.0-11.5)$ & 85 & $5.0(4.0-11.0)$ & 15 & $5.0(4.0-139.0)$ & 66 & $5.0(4.0-13.0)$ & 34 & $5.0(4.0-10.0)$ \\
\hline Non transferrin bound iron $(\mu \mathrm{mol} / \mathrm{L})$ & 100 & $0.7(0.1-3.0)$ & 85 & $0.6(0.1-2.9)$ & 15 & $1.0(0.1-3.4)$ & 66 & $0.5(0.1-1.8)$ & 34 & $1.2(0.3-3.8)$ \\
\hline Labile plasma iron $(\mu \mathrm{mol} / \mathrm{L})$ & 100 & $0.1(0.0-0.2)$ & 85 & $0.1(0.0-0.2)$ & 15 & $0.1(0.0-0.3)$ & 66 & $0.1(0.0-0.2)$ & 34 & $0.1(0.0-0.3)$ \\
\hline $\begin{array}{l}\text { Growth differentiation } \\
\text { factor } 15(\mathrm{ng} / \mathrm{L})\end{array}$ & 100 & $\begin{array}{c}2193 \\
(952-5663)\end{array}$ & 85 & $\begin{array}{c}2140 \\
(921-6084)\end{array}$ & 15 & $\begin{array}{c}2823 \\
(1232-5026)\end{array}$ & 66 & $\begin{array}{c}1844 \\
(921-4828)\end{array}$ & 34 & $\begin{array}{c}2888 \\
(1026-10361)\end{array}$ \\
\hline
\end{tabular}


dromes, and levels are suppressed in case of decreased erythropoietic activity, as in anemia of chronic disease, and diseases with erythroid hypoplasia. ${ }^{20,25,28}$ Earlier studies showed that sTFR levels are increased in MDS-RS, ${ }^{5}$ including SF3B1-positive MDS patients. ${ }^{11}$

NTBI concentrations are only sporadically present with transferrin saturations (TSAT) $<70 \%$ and increase sharply when the saturation of transferrin with iron exceeds $70 \% .{ }^{29}$ Chemically, NTBI consists of iron that is rather loosely bound to albumin or low molecular weight metal complexing groups. ${ }^{30,31}$ The NTBI complexes may be taken up by specific NTBI transporters in the liver, pancreas, and heart and contribute to oxidant-mediated cellular injury in these tissues. ${ }^{17,32}$ LPI is thought to be the NTBI fraction that is mostly responsible for tissue injury, since it is readily available to participate in redox cycling, causing oxidative damage to cellular membranes, proteins and DNA. ${ }^{15,33}$ It has been proposed that plasma NTBI is an important early indicator of extra-hepatic iron toxicity in $\beta$-thalassemia major. ${ }^{34,35}$

Improved insights in the levels and roles of key players of iron metabolism during treatment with transfusions in the various $\mathrm{MDS}$ subtypes may provide leads for novel diagnostic and iron reducing treatment strategies. The prospective study of the EUMDS registry was initiated in order to provide a better understanding of the pathophysiology and prognostic value of iron overload and ironmediated oxidative stress as well as possibly important markers in iron homeostasis over time in MDS. To this end, we evaluated serum ferritin, iron, transferrin saturation, hepcidin-25, GDF15, sTFR, NTBI and LPI levels over time in lower-risk MDS patients and their relation with regard to the World Health Organization (WHO) 2001 subtype and transfusion history. We identified detectable LPI levels as a new important prognostic factor for survival in patients with MDS-RS or lower-risk MDS patients treated with regular $\mathrm{RBC}$ transfusions.

\section{Methods}

\section{Study design and participants}

Patients were eligible to be included in the EUMDS registry if they were newly diagnosed with MDS according to the WHO 2001 classification and a low or intermediate- 1 score according to the International Prognostic Scoring System (IPSS). Two patients with IPSS intermediate-2 or high-risk patients with secondary or therapyrelated MDS were excluded from this registry. The ethics committees of all participating countries and centers approved the protocol. Patients were required to provide written informed consent.

Serum samples were collected prospectively, at registration and at 6-month intervals, from 109 patients from six countries who participated in this study from April 2008 to December 2010 Samples from nine patients had to be excluded due to technical reasons, see Online Supplementary Information for details. The total number of analyzed serum samples was 454 .
A

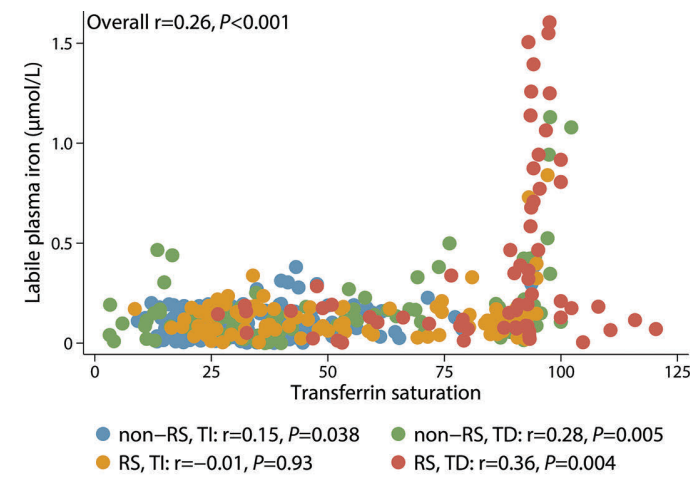

B

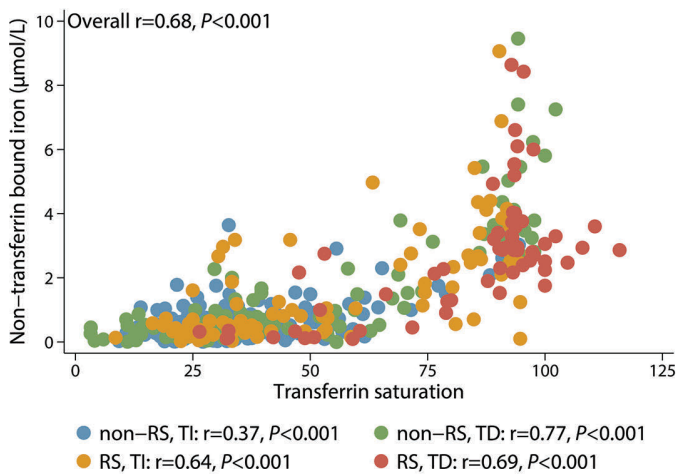

C

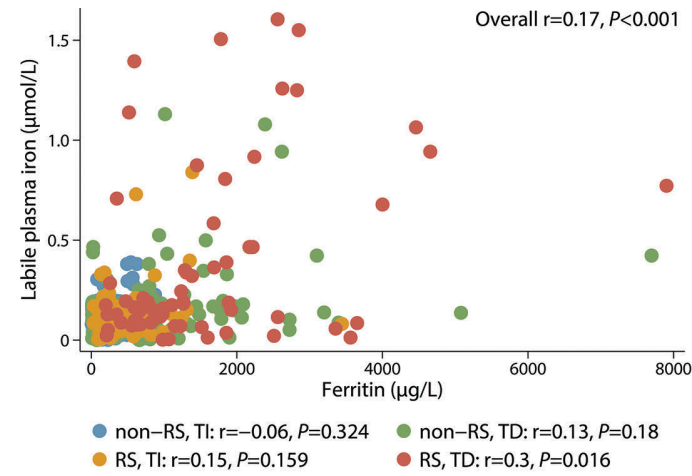

D

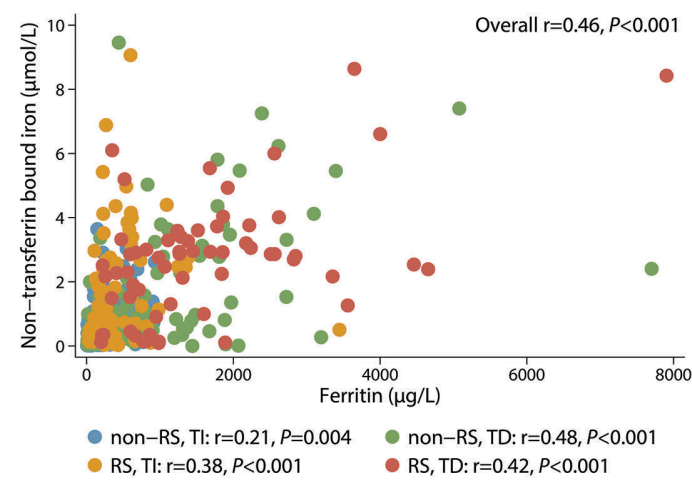

Figure 1. LPI and NTBI correlated to TSAT and ferritin in different patient groups. (A) Relation between LPI and TSAT. (B) Relation between NTBI and TSAT. (C) Relation between LPI and ferritin. (D) Relation between NTBI and ferritin. Each dot represents one sample (median: 5 samples/patient). RS: ring sideroblastic; TI: transfusion-independent; TD: transfusion-dependent. 


\section{Biochemical assays}

The iron parameters in this substudy were analyzed centrally at the department of Laboratory Medicine of the Radboudumc, Nijmegen, The Netherlands. Detailed information regarding these iron parameters is described in the Online Supplementary Information.

Measurement of serum NTBI consisted of the chelation-ultrafiltration-detection approach based on the prior mobilization of serum NTBI by weak iron-mobilizing chelators, such as nitrilotriacetate (NTA), at $80 \mathrm{mM}$. The chelated NTBI was separated from transferrin-bound iron by ultrafiltration and detected by colorimetry. ${ }^{36}$ The lower limit of detection (LLOD) of the NTBI assay was $0.47 \mu \mathrm{mol} / \mathrm{L}$. The LPI measurement was based on the measurement of the redox-active and readily chelatable fraction of NTBI. This assay measures iron-catalyzed radical generation in the presence of a low ascorbate concentration. Radical generation was measured with the fluorogenic redox sensitive probe dihydrorhodamine (DHR) 123, and iron-catalyzed radical generation was calculated by subtracting the radical generation in the presence of 50 $\mu \mathrm{mol} / \mathrm{L}$ of the bidendate iron chelator deferiprone (DFO, the LPI DHR oxidation that is NOT iron dependent). ${ }^{37}$ The LLOD of the LPI assay was $0.24 \mu \mathrm{mol} / \mathrm{L}$.

\section{Statistical analysis}

Standard descriptive techniques were used to assess the association between the iron parameters including Spearman's rank correlation coefficients. Where NTBI or LPI was below LLOD, values were randomly drawn from a univariate distribution in the range from zero to the LLOD. Overall survival (OS) was defined as the time from date of diagnosis to death, or for subjects still alive and censored, to the date of the last visit when a sample was available. Cox proportional hazards regression models and Kaplan-Meier survival curves with time-dependent covariates ${ }^{38}$ were used in time-to-event analyses to assess the impact of LPI levels, NTBI and TSAT by transfusion status on survival. All variables were treated as time-varying covariates in the model by assessing the levels of the parameters (LPI, NTBI: $<$ LLOD vs. elevated, TSAT $<80 \%$ vs. $\geq 80 \%$ ) and transfusion status (transfused $v s$. not transfused) at each visit. LPI and NTBI levels $>$ LLOD were considered abnormal. Once a subject had received a transfusion, they were classified as transfused for the remaining time. Hazard ratios (HR) and 95\% confidence intervals $(95 \% \mathrm{CI})$ are reported for both univariate and multivariate models. In the case of multivariate analyses, the additional covariates included were age at diagnosis, IPPS-revised (IPSS-R) category and usage of erythroid stimulating agents (ESA). All analyses were undertaken in Stata 14 (StataCorp, College Station, TX, USA).

\section{Results}

\section{Patient characteristics}

The median age of all patients at registration was 73 years (range: $43-95$ years). The majority of the patients were male; $64 \%(n=64)$. The IPSS risk groups of the 100 patients in the study were: low $47 \%$, intermediate- $141 \%$, and unknown $12 \%$. The IPSS-R risk groups were: very low $32 \%$, low $41 \%$, intermediate $8 \%$, high $3 \%$, and unknown $16 \%$. WHO 2001 MDS-subtypes were refractory cytopenia with multilineage dysplasia (RCMD; 37\%), refractory anemia with ring sideroblasts (RARS; $30 \%$ ), refractory anemia (RA; 18\%), refractory anemia with excess blasts (RAEB; 7\%), 5q-syndrome (4\%) and refractory cytopenia with multilineage dysplasia and ringed sideroblasts (RCMD-RS; 4\%). Fourteen percent of the patients were transfusion-dependent at registration $(n=14)$. No patients received iron chelation therapy at the time of registration. Six patients received iron chelation therapy during this observation period (Online Supplementary Table S1). The median number of samples available per patient was 5 (range: 1-7), and the median follow-up period was 5.8 years. OS and progression-free survival (PFS) in our study population were 4.8 and 4.6 years, respectively. Nineteen patients died, including 5 patients after progression and 9 patients from causes possibly related to MDS (hemorrhage 2 , infection 5, and cardiovascular 2) (Online Supplementary Table S2).

\section{Iron parameters}

Median ferritin levels were elevated $(>250 \mu \mathrm{g} / \mathrm{l})$ at registration in all patient groups, but the highest median levels were observed in the transfusion-dependent (TD) groups (Table 1). Median serum iron levels were within reference range $(12-30 \mu \mathrm{mol} / \mathrm{L})$ in all patient groups at registration. Overall, median TSAT was within reference range $(<45 \%)$ at registration, with the exception of TD MDS-RS patients (Table 2). Median hepcidin levels were within reference range in all patient groups at registration, but TD patients had significantly higher hepcidin levels compared to transfusion-independent (TI) patients $(P<0.001)$. Ferritin levels correlated significantly with hepcidin levels $(r=0.55$,
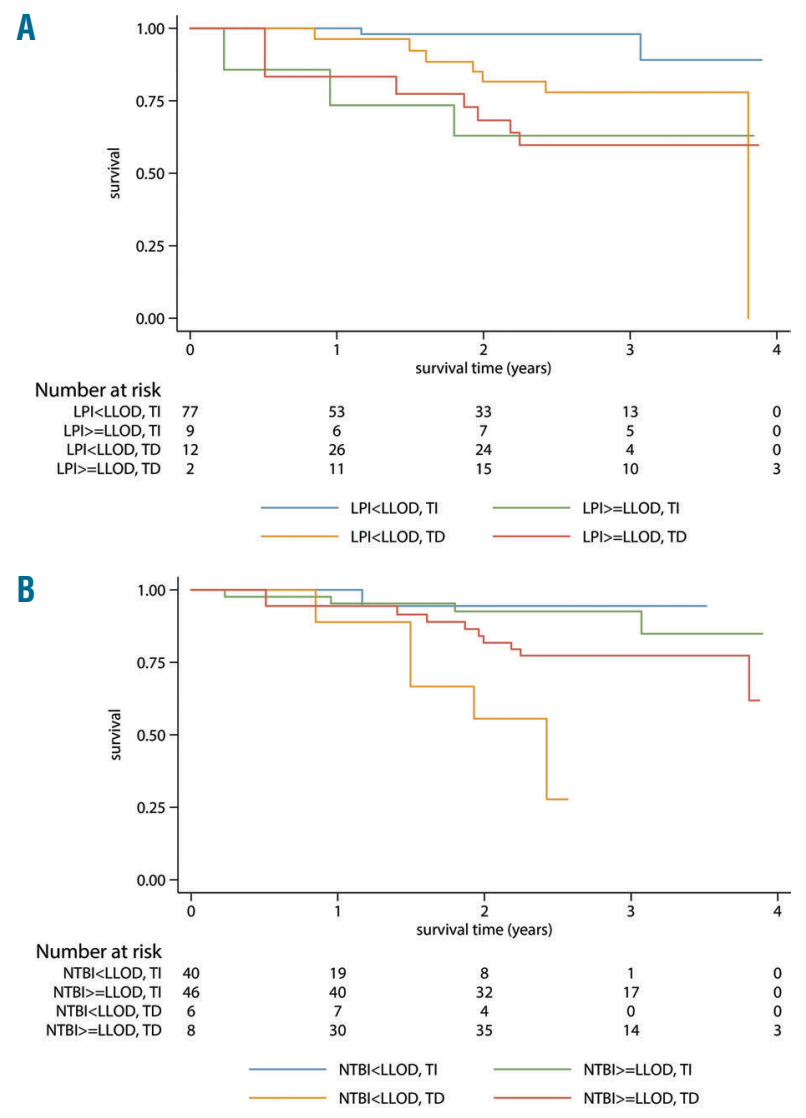

Figure 2 Survival according to LPI (A) or NTBI (B) and transfusion status. LPI, NTBI and transfusion status were analyzed as time dependent factors, implicating that patients may switch groups over time according to the LPI/NTBI and transfusion status at each specific time point. LLOD: lower limit of detection; TI: transfusion-independent; TD: transfusion-dependent; LPI: labile plasma iron; NTBI: non-transferrin bound iron. 
$P<0.001)$. The median GDF15 levels were elevated in the RS subgroup only. NTBI levels above LLOD ( $>0.47$ $\mu \mathrm{mol} / \mathrm{L}$ ) occurred in all patient groups at registration with the highest levels present in MDS-RS patients. sTFR levels were within the reference range $(0.8-1.8 \mathrm{mg} / \mathrm{L})$ at registration, and the highest levels were observed in TI MDS-RS patients (Table 2). The median LPI levels were below LLOD in all patient groups at registration $(<0.24 \mathrm{~mol} / \mathrm{L})$, except in TD MDS-RS patients. Median C-reactive protein (CRP) levels were below the upper limit of the reference range $(<10 \mathrm{mg} / \mathrm{L})$ in all groups at all time points (Table 1 ) and the majority of patients with CRP levels above 50 $\mathrm{mg} / \mathrm{L}$ were TD. CRP levels correlated positively with hep- cidin levels $(r=0.30, P<0.001)$ and ferritin levels $(r=0.22$, $P<0.001)$.

\section{Impact of MDS subtype and transfusions on iron parameters over time}

The impact of transfusions and MDS subtype (RS vs. non-RS) on TSAT, hepcidin, GDF15, NTBI and LPI levels over time is shown in Table 2. Both serum ferritin and serum iron levels increased significantly $(r=0.59, P<0.001$ and $r=0.32, P<0.001$, respectively) with a cumulative number of transfused units over time in TD patients (Online Supplementary Table S3) as well as in RS patients (Online Supplementary Table S4). TSAT remained stable and within

Table 2. Frequency, median and quartiles of iron parameters by transfusion status per MDS subtype at registration, 1 year and 2 years follow-up.

\begin{tabular}{|c|c|c|c|c|c|c|}
\hline & N & $\begin{array}{c}\text { Registration } \\
\text { Median(p10-p90) }\end{array}$ & N & $\begin{array}{l}1 \text { year follow-up } \\
\text { Median(p10-p90) }\end{array}$ & N & $\begin{array}{l}2 \text { years follow-up } \\
\text { Median(p10-p90) }\end{array}$ \\
\hline \multicolumn{7}{|l|}{ Transferrin } \\
\hline saturation (\%) & 100 & $35.6(19.0-87.4)$ & 78 & $34.4(16.4-92.9)$ & 64 & $37.5(22.2-94.3)$ \\
\hline MDS non-RS: TI & 56 & $32.8(17.1-55.6)$ & 32 & $28.4(17.4-59.1)$ & 26 & $30.1(18.8-54.2)$ \\
\hline MDS non-RS: TD & 10 & $28.7(8.5-77.9)$ & 21 & $36.8(14.0-89.1)$ & 17 & $39.3(20.4-97.7)$ \\
\hline MDS-RS: TI & 29 & $48.8(24.6-92.5)$ & 16 & $36.4(20.8-86.4)$ & 9 & $35.6(23.9-92.6)$ \\
\hline MDS-RS: TD & 5 & $90.0(53.1-120.4)$ & 9 & $93.6(42.1-110.6)$ & 12 & $93.1(71.7-97.6)$ \\
\hline Hepcidin (nmol/L) & 99 & $4.5(1.1-21.7)$ & 78 & $5.6(1.2-19.6)$ & 65 & $5.2(1.0-19.6)$ \\
\hline MDS non-RS: TI & 56 & $4.5(1.7-22.1)$ & 32 & $4.3(1.5-11.8)$ & 26 & $4.6(0.9-13.6)$ \\
\hline MDS non-RS: TD & 10 & $4.9(0.5-75.9)$ & 21 & $17.3(0.5-29.2)$ & 17 & $9.2(1.3-28.4)$ \\
\hline MDS-RS: TI & 28 & $3.8(1.0-8.7)$ & 16 & $3.4(0.5-5.8)$ & 9 & $2.9(0.8-12.2)$ \\
\hline MDS-RS: TD & 5 & $10.3(3.8-15.9)$ & 9 & $9.2(3.8-14.4)$ & 13 & $5.2(1.0-14.6)$ \\
\hline \multicolumn{7}{|l|}{ Growth differentiation } \\
\hline factor 15 (ng/L) & 100 & $2193(952-5663)$ & 77 & $2479(1016$ - 7982) & 63 & $2576(1045-7746)$ \\
\hline MDS non-RS: TI & 56 & $1777(731-4658)$ & 32 & $1653(615-5684)$ & 26 & $1685(633-5736)$ \\
\hline MDS non-RS: TD & 10 & 2306 (1218 - 4927) & 20 & $2583(1725$ - 7166) & 17 & $2998(1398$ - 8037) \\
\hline MDS-RS: TI & 29 & 2619 (996 - 11083) & 16 & 2694 (1223 - 10303) & 8 & $2780(1331-9554)$ \\
\hline MDS-RS: TD & 5 & $2893(2113$ - 5370) & 9 & $3866(830$ - 15167) & 12 & $5361(1053-8399)$ \\
\hline \multicolumn{7}{|l|}{ Soluble transferrin } \\
\hline receptor (mg/L) & 100 & $1.3(0.7-2.8)$ & 78 & $1.4(0.7-3.0)$ & 62 & $1.3(0.8-2.7)$ \\
\hline MDS non-RS: TI & 56 & $1.2(0.8-2.7)$ & 32 & $1.4(0.9-2.8)$ & 26 & $1.2(0.9-2.7)$ \\
\hline MDS non-RS: TD & 10 & $1.0(0.6-2.8)$ & 21 & $1.1(0.4-3.1)$ & 16 & $1.2(0.6-2.2)$ \\
\hline MDS-RS: TI & 29 & $1.6(0.8-3.3)$ & 16 & $2.0(1.1-2.8)$ & 8 & $2.2(1.0-2.8)$ \\
\hline MDS-RS: TD & 5 & $0.9(0.4-3.1)$ & 9 & $1.2(0.6-3.1)$ & 12 & $1.4(0.4-3.6)$ \\
\hline \multicolumn{7}{|l|}{ Non transferrin } \\
\hline bound iron $(\mu \mathrm{mol} / \mathrm{L})$ & 100 & $0.65(0.14-3.03)$ & 77 & $0.59(0.15-3.64)$ & 65 & $0.64(0.14-5.42)$ \\
\hline MDS non-RS: TI & 56 & $0.41(0.10-1.51)$ & 31 & $0.42(0.03-0.91)$ & 26 & $0.50(0.18-1.78)$ \\
\hline MDS non-RS: TD & 10 & $0.80(0.05-2.73)$ & 21 & $0.69(0.16-3.64)$ & 17 & $1.00(0.12-7.25)$ \\
\hline MDS-RS: TI & 29 & $0.88(0.26-3.99)$ & 16 & $0.70(0.16-3.52)$ & 9 & $0.52(0.05-5.42)$ \\
\hline MDS-RS: TD & 5 & $3.03(1.90-3.40)$ & 9 & $3.60(0.15-8.64)$ & 13 & $2.86(0.46-7.57)$ \\
\hline \multicolumn{7}{|l|}{ Labile plasma } \\
\hline iron $(\mu \mathrm{mol} / \mathrm{L})$ & 100 & $0.09(0.02-0.22)$ & 77 & $0.13(0.03-0.38)$ & 65 & $0.13(0.02-0.38)$ \\
\hline MDS non-RS: TI & 56 & $0.10(0.03-0.19)$ & 31 & $0.10(0.02-0.17)$ & 26 & $0.11(0.01-0.30)$ \\
\hline MDS non-RS: TD & 10 & $0.06(0.01-0.18)$ & 21 & $0.17(0.06-0.38)$ & 17 & $0.14(0.02-1.08)$ \\
\hline MDS-RS: TI & 29 & $0.10(0.02-0.32)$ & 16 & $0.09(0.05-0.24)$ & 9 & $0.10(0.03-0.17)$ \\
\hline MDS-RS: TD & 5 & $0.08(0.00-0.35)$ & 9 & $0.47(0.06-1.26)$ & 13 & $0.19(0.08-1.39)$ \\
\hline
\end{tabular}

MDS: Myelodysplastic syndromes; RS: ring sideroblastic; TI: transfusion-independent; TD: transfusion-dependent. 
reference range in the TI patients, with the exception of a minority of RS patients (Online Supplementary Figure S1), and increased over time in the TD patients, with up to $94.9 \%$ in patients with $>10 \mathrm{RBC}$ units transfused (Online Supplementary Table S3). Hepcidin levels increased with the number of units transfused; in contrast, hepcidin levels significantly decreased over time in TI MDS-RS patients (Online Supplementary Table S4). GDF15 levels were not associated with transfusion status alone, but did increase over time in TD MDS-RS patients with a median of 2893 $\mathrm{ng} / \mathrm{L}$ at registration compared to $5361 \mathrm{ng} / \mathrm{L}$ at 2 years follow up.

STFR levels increased significantly $(P<0.001)$ over time in both TI and TD MDS-RS patients $(P=0.01)$ (Table 2). STFR levels did not change over time in non-RS MDS patients. The lowest sTFR levels were observed in patients who had received more than 10 units (Online Supplementary Table S3). TD MDS-RS patients had the most elevated levels of NTBI and LPI over time (Online Supplementary Table S3 and S4).

\section{Correlation between markers of iron overload}

Both elevated NTBI and LPI levels (>LLOD) showed a threshold effect with TSAT of $>70 \%$ and $>80 \%$, respectively (Figure 1A,B). Detectable LPI levels occurred almost exclusively in patients with MDS-RS and/or patients who had received transfusions. NTBI and LPI levels above the LLOD were mutually positively correlated $(r=0.46$; $P<0.001)$. Both NTBI and LPI showed a linear relationship $(P<0.001)$ with ferritin, but no threshold levels could be detected (Figure 1C,D). The highest values were observed in TD MDS patients; subgroup analyses showed mainly a positive correlation in the TD and/or RS subgroup (Figure $1 C, D)$.

\section{Prognostic impact of iron overload markers}

Time-dependent, multivariate analysis of overall survival, adjusted for age and IPSS-R risk groups revealed no significant effect on overall survival for NTBI ( $\mathrm{HR}=0.56$, $95 \% \mathrm{CI} 0.21-1.52 ; P=0.26)$ and for TSAT (HR=0.91, 95\%CI 0.29-2.86; $P=0.88$ ) (Table 3, Figure $2 \mathrm{~B}$ and Online Supplementary Figure S1).

Ten out of 19 patients who died during this study had detectable LPI. The majority (7 patients) died from progression or MDS-related causes (Online Supplementary Table S2). Kaplan-Meier curves demonstrate prognostic impact on survival of detectable LPI levels by transfusion status (Figure 2), but no significant effect in the multivariate analysis adjusted for age and IPSS-R risk ( $\mathrm{HR}=2.1$, 95\%CI 0.7-6.2; Table 3). Once LPI was increased in both TD and TI patients, survival time decreased, with the greatest impact observed in patients who were TD and had increased LPI levels (adjusted HR=3.0, 95\%CI 0.713.3). Since 41 patients were also treated with erythropoietin stimulating agents (ESA), we repeated the analyses adjusted for whether or not the patient had been treated with ESA at each visit (Figure 3). These adjustments did not significantly alter the magnitude of the risk estimates on OS ( $\mathrm{HR}=3.0,95 \%$ CI 0.7-13.5) (Table 3).

Because the survival of patients with RS-MDS is usually considered better than in the non-RS MDS population, we repeated the analyses in the largest group of 66 non-RS

Table 3. Cox model of OS by labile plasma iron, non-transferrin bound iron and transferrin saturation along with transfusion status as time varying variable for all patients $(n=100)$.

\begin{tabular}{|c|c|c|c|c|c|c|c|c|}
\hline & \multicolumn{3}{|c|}{ Unadjusted } & \multirow{2}{*}{$\begin{array}{c}\text { Adjusted }^{1} \\
\text { P }\end{array}$} & \multicolumn{2}{|c|}{ Adjusted ${ }^{2}$} & \multirow{2}{*}{\multicolumn{2}{|c|}{$\begin{array}{cc}\text { Adjusted }^{3} \\
\text { io } & P\end{array}$}} \\
\hline & $\begin{array}{l}\text { Hazard ratio } \\
\text { (95\% Cl) }\end{array}$ & $\mathbf{P}$ & $\begin{array}{l}\text { Hazard ratio } \\
(95 \% \text { CI) }\end{array}$ & & $\begin{array}{l}\text { Hazard ratio } \\
(95 \% \text { Cl) }\end{array}$ & $\mathbf{P}$ & & \\
\hline LPI $(\mu \mathrm{mol} / \mathrm{L})<\mathrm{LLOD}$ & 1 & - & 1 & - & 1 & - & 1 & - \\
\hline$\geq$ LLOD & $2.2(0.8-6.2)$ & 0.14 & $2.0(0.7-6.0)$ & 0.21 & $2.0(0.7-5.8)$ & 0.23 & $2.0(0.7-6.2)$ & 0.20 \\
\hline LPI<LLOD, TI & 1 & - & 1 & - & 1 & - & 1 & - \\
\hline LPI $\geq L L O D, T I$ & $4.6(0.5-42.4)$ & 0.18 & $3.2(0.3-30.2)$ & 0.31 & $3.3(0.4-31.1)$ & 0.30 & $3.2(0.3-30.4)$ & 0.31 \\
\hline LPI <LLOD, TD & $4.1(1.2-13.6)$ & 0.02 & $2.0(0.5-7.1)$ & 0.30 & $2.2(0.6-8.1)$ & 0.24 & $2.0(0.5-7.1)$ & 0.31 \\
\hline $\mathrm{LPI} \geq \mathrm{LLOD}, \mathrm{TD}$ & $4.7(1.1-19.7)$ & 0.03 & $3.0(0.7-13.3)$ & 0.15 & $3.0(0.7-13.5)$ & 0.14 & $3.0(0.7-13.4)$ & 0.15 \\
\hline NTBI $(\mu \mathrm{mol} / \mathrm{L})<\mathrm{LLOD}$ & 1 & - & 1 & - & 1 & - & 1 & - \\
\hline$\geq$ LLOD & $0.7(0.3-1.7)$ & 0.39 & $0.6(0.2-1.6)$ & 0.27 & $0.5(0.2-1.5)$ & 0.24 & $0.6(0.2-1.5)$ & 0.26 \\
\hline NTBI<LLOD, TI & 1 & - & 1 & - & 1 & - & 1 & - \\
\hline NTBI $\geq L L O D, T I$ & $0.6(0.1-3.8)$ & 0.61 & $0.7(0.1-4.0)$ & 0.65 & $0.7(0.1-4.2)$ & 0.67 & $0.6(0.1-4.0)$ & 0.62 \\
\hline NTBI<LLOD, TD & $4.7(1.1-19.0)$ & 0.03 & $2.6(0.6-11.6)$ & 0.22 & $3.1(0.7-14.4)$ & 0.14 & $2.5(0.6-11.5)$ & 0.22 \\
\hline NTBI $\geq L L O D, T D$ & $2.2(0.5-8.6)$ & 0.27 & $1.1(0.3-5.0)$ & 0.86 & $1.2(0.3-5.4)$ & 0.80 & $1.1(0.3-4.9)$ & 0.89 \\
\hline TSAT < <80\% & 1 & - & 1 & - & 1 & - & 1 & - \\
\hline$>80 \%$ & $1.3(0.4-3.6)$ & 0.66 & $0.9(0.3-2.9)$ & 0.88 & $0.9(0.3-2.8)$ & 0.85 & $1.0(0.3-3.1)$ & 0.97 \\
\hline TSAT <80\%, TI & 1 & - & 1 & - & 1 & - & 1 & - \\
\hline TSAT $\geq 80 \%$, TI & $2.5(1.0-6.2)$ & 0.04 & $2.3(0.9-5.7)$ & 0.08 & $2.5(1.0-6.5)$ & 0.05 & $2.3(0.9-5.9)$ & 0.10 \\
\hline TSAT < $80 \%$, TD & $1.9(1.2-3.0)$ & 0.003 & $1.6(1.0-2.5)$ & 0.05 & $1.7(1.1-2.7)$ & 0.03 & $1.6(0.99-2.5)$ & 0.053 \\
\hline $\mathrm{TSAT} \geq 80 \%, \mathrm{TD}$ & $1.3(0.9-2.0)$ & 0.19 & $1.1(0.7-1.7)$ & 0.70 & $1.1(0.7-1.7)$ & 0.67 & $1.1(0.7-1.7)$ & 0.70 \\
\hline
\end{tabular}

${ }^{1}$ Adjusted for age at diagnosis and IPSS-R ${ }^{2}$ Adjusted for age at diagnosis, IPSS-R and ESA treatment status at each visit. ${ }^{3}$ Adjusted for age,IPSS-R and RS status. CI: confidence interval; LLOD: lowest level of detection; LPI: labile plasma iron;TI: transfusion-independent; TD: transfusion-dependent; NTBI: non-transferrin bound iron; TSAT: transferrin saturation. 
patients (Table 4). Detectable LPI levels had a remarkable impact on survival in the whole non-RS group, but the impact was only significant in the TD subgroup ( $\mathrm{HR}=17.0$, 95\% CI 2.0-146.6). TSAT levels had a borderline impact on survival in TI patients.

Six patients received iron chelation in this study (Online Supplementary Table S1). LPI levels during treatment with deferasirox decreased below LLOD (4 patients), even in those patients with high TSAT. Only 3 patients were treated with lenalidomide.

Ferritin levels and elevated CRP are time-dependent variables, which correlate closely with transfusion burden/transfusion intensity, and presumably with infections (data not shown). Ferritin levels and elevated CRP predict survival when adjusted for age and IPSS-R group only, but the prognostic impact is less clear when transfusion intensity was added to the model (data not shown).

\section{Discussion}

This study among 100 European lower-risk MDS patients showed that both RBC transfusions and the presence of RS increased the occurrence of the toxic iron species NTBI and LPI in serum. Our data on iron parameters over time suggest that body iron accumulation and

Table 4. Cox model of OS by labile plasma iron, non-transferrin bound iron and transferrin saturation along with transfusion status as time varying variable for non-RS patients only $(n=66)$.

\begin{tabular}{|c|c|c|c|c|c|c|}
\hline & $\begin{array}{l}\text { Unadjusted } \\
\text { Hazard ratio } \\
\text { (95\% CI) }\end{array}$ & $P$ & $\begin{array}{l}\text { Adjusted }^{1} \\
\text { Hazard ratio } \\
\text { (95\% CI) }\end{array}$ & $P$ & $\begin{array}{l}\text { Adjusted }^{2} \\
\text { Hazard ratio } \\
\text { (95\% CI) }\end{array}$ & $P$ \\
\hline LPI $(\mu \mathrm{mol} / \mathrm{L})<\mathrm{LLOD}$ & 1 & - & 1 & - & 1 & - \\
\hline Elevated & $4.9(1.4-16.8)$ & 0.01 & $5.4(1.5-19.6)$ & 0.01 & $9.3(2.0-43.3)$ & 0.004 \\
\hline LPI $<$ LLOD, TI & 1 & - & 1 & - & 1 & - \\
\hline LPI $\geq$ LLOD, TI & $10.2(0.9-115.4)$ & 0.06 & $5.3(0.4-68.9)$ & 0.20 & $5.9(0.4-86.2)$ & 0.19 \\
\hline LPI <LLOD, TD & $4.6(0.9-23.5)$ & 0.07 & $2.0(0.3-12.0)$ & 0.47 & $1.4(0.2-8.9)$ & 0.70 \\
\hline LPI $\geq$ LLOD, TD & $11.8(1.9-74.0)$ & 0.008 & $10.3(1.3-79.5)$ & 0.03 & $17.0(2.0-146.6)$ & 0.01 \\
\hline NTBI $(\mu \mathrm{mol} / \mathrm{L})<\mathrm{LLOD}$ & 1 & - & 1 & - & 1 & - \\
\hline Elevated & $0.6(0.2-1.9)$ & 0.37 & $0.6(0.2-2.0)$ & 0.38 & $0.6(0.2-2.2)$ & 0.46 \\
\hline NTBI<LLOD, TI & 1 & - & 1 & - & 1 & - \\
\hline NTBI $\geq L L O D, T I$ & $0.6(0.1-6.9)$ & 0.70 & $1.1(0.1-14.4)$ & 0.92 & $1.1(0.09-14.3)$ & 0.92 \\
\hline NTBI<LLOD, TD & $5.7(1.1-30.3)$ & 0.04 & $5.7(0.8-42.2)$ & 0.09 & $5.4(0.7-43.7)$ & 0.11 \\
\hline NTBI $\geq L L O D, T D$ & $2.1(0.4-12.3)$ & 0.39 & $1.4(0.2-8.2)$ & 0.74 & $1.4(0.2-8.2)$ & 0.74 \\
\hline TSAT $<80$ & 1 & - & 1 & - & 1 & - \\
\hline Elevated & $2.1(0.6-7.8)$ & 0.28 & $1.1(0.2-5.4)$ & 0.90 & $1.5(0.3-8.6)$ & 0.63 \\
\hline TSAT $<80$, TI & 1 & - & 1 & - & 1 & - \\
\hline $\mathrm{TSAT} \geq 80$, TI & $3.8(1.1-12.7)$ & 0.03 & $3.7(0.98-13.8)$ & 0.053 & $3.7(0.99-14.1)$ & 0.052 \\
\hline TSAT <80, TD & $1.9(1.1-3.2)$ & 0.02 & $1.7(0.9-3.2)$ & 0.13 & $1.6(0.8-3.2)$ & 0.21 \\
\hline $\mathrm{TSAT} \geq 80$, TD & $1.5(0.9-2.5)$ & 0.12 & $1.1(0.6-1.9)$ & 0.80 & $1.1(0.6-1.9)$ & 0.77 \\
\hline
\end{tabular}

${ }^{1}$ Adjusted for age at diagnosis and IPSS-R. ${ }^{2}$ Adjusted for age at diagnosis, IPSS-R and ESA treatment status at each visit. LLOD: lowest level of detection; LPI: labile plasma iron; TI: transfusion-independent; TD: transfusion-dependent; TSAT: transferrin saturation; CI: confidence interval.

Total number study patients

Any transfusions

ESA treatment

Timing of ESA treatment

\section{Stopped transfusions after starting ESA}

\section{Subsequently restarted transfusions}
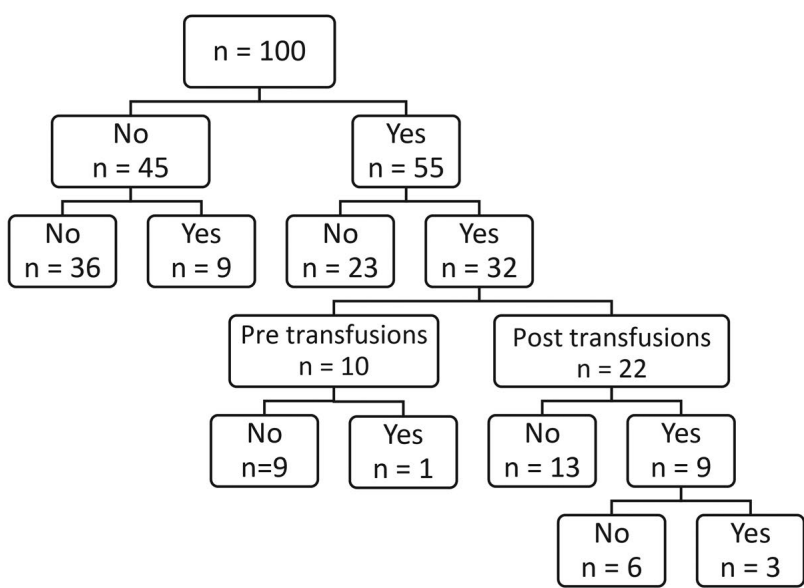

Figure 3 Flow diagram of patients treated with transfusions and erythropoietin stimulating agents (ESAs). In total, 10 patients became transfusion-independent after starting ESA treatment 
toxic iron species (NTBI and LPI) in RS-MDS patients occur along the axis of ineffective erythropoiesis, characterized by elevated sTFR, increased GDF15, low hepcidin, and increased circulating and parenchymal iron levels (Figure 4A). Interestingly we found detectable LPI, but not
NTBI, to be associated with a significantly decreased OS in non-sideroblastic MDS patients.

Hepcidin levels were significantly elevated in all TD patient categories immediately after the initiation of transfusions, and remained elevated during transfusion

A

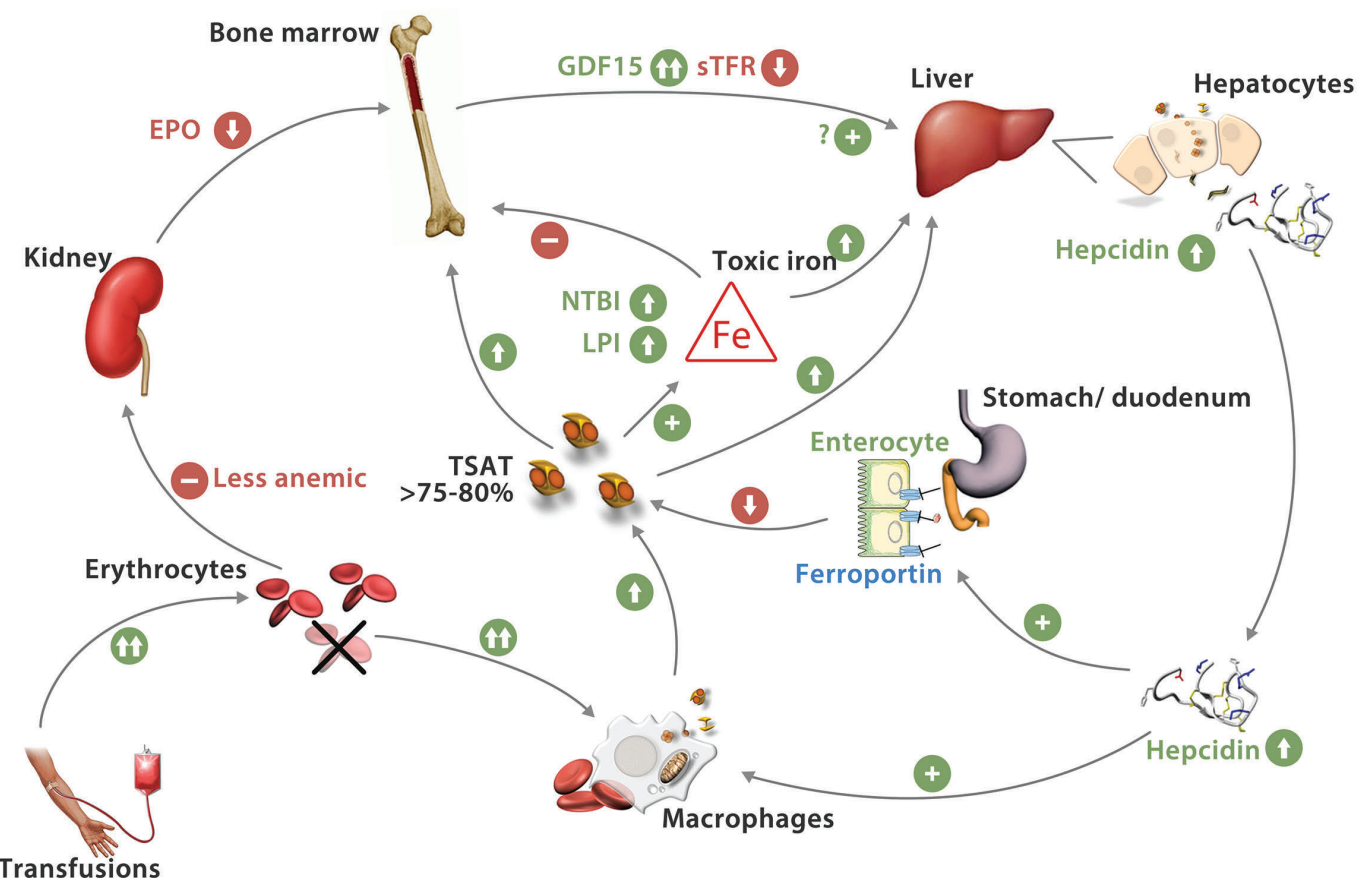

B

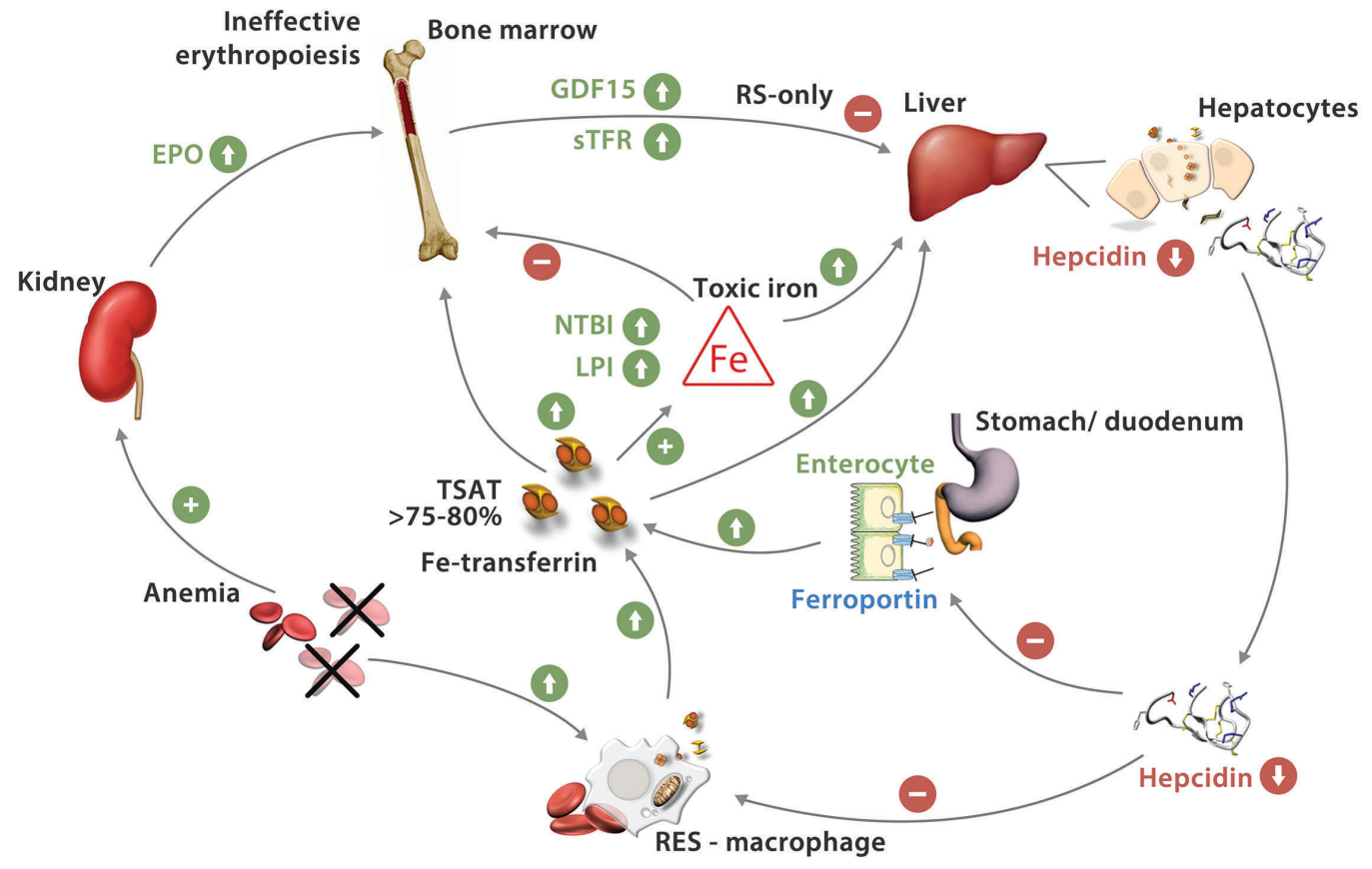

Figure 4. Proposed pathogenesis of iron toxicity in lower-risk MDS: the impact of ineffective erythropoiesis (A) and of transfusions (B). Ineffective erythropoiesis , especially in RS MDS, results in increased bone marrow production of GDF15 and possibly twisted gastrulation 1 and erythroferrone. These factors inhibit hepcidin production by the hepatocytes. Low hepcidin levels increase iron absorption from intestinal mucosa and increase iron release from the macrophages. Eventually, this may lead to toxic levels of NTBI and LPI, causing damage in solid organs, the immune system and the marrow. During transfusions hepcidin levels increase, despite higher GDF15 levels, leading to lower iron absorption in the gut. However, transfusions cause massive iron loading of RES-macrophages leading to elevated, circulating stored iron levels and toxic iron species - despite elevated hepcidin levels - and subsequent toxicities. Figure adapted from ML Cuijpers, et al. ${ }^{6}$ RS: ring sideroblastic; GDF15: growth differentiation factor 15; TWSG1: twisted gastrulation 1; LPI: labile plasma iron; NTBI: non-transferrin bound iron; sTFR: soluble transferrin receptor; RES: reticuloendothelial system; TSAT: transferrin saturations; EPO: erythropoietin. 
dependency, confirming recent studies in transfused MDS patients and illustrated in Figure 4B. ${ }^{5,7}$ However, the elevated hepcidin levels showed a tendency to decrease during continued exposure to transfusions. In addition, sTFR levels decreased over time in TD patients, compatible with previously reported suppression of erythropoiesis by continued transfusions. ${ }^{20,25}$ Interestingly, GDF15 increased over time in TD MDS patients and especially in those categorized as TD RS-MDS. Increased GDF15 has previously been associated with ineffective erythropoiesis, but not with TD-mediated suppression of erythropoiesis. ${ }^{5}$ This suggests that TD-mediated suppression of ineffective erythropoiesis may be less effective during prolonged transfusions. This is supported by the gradual decline over time of the initially elevated hepcidin levels during prolonged transfusions. These data show that previous conflicting observations on the relationship of GDF15 and hepcidin can be explained by the impact of transfusions on GDF15 and hepcidin levels, especially in RS-MDS patients. ${ }^{5}$

Hepcidin levels decreased over time in TI patients of the RS subtype. An earlier study in 107 untransfused patients observed generally elevated hepcidin levels in MDS, but low hepcidin/ferritin ratios in the RS subtypes, compatible with the low hepcidin levels in the RS patients of our study. ${ }^{39}$ In addition, RS patients showed elevated sTFR levels and decreased hepcidin levels compared to TI non-RS at all time points. These observations confirm the previously reported association between sTFR and ineffective erythropoiesis, resulting in an increased uptake of dietary iron and iron release by macrophages, subsequently leading to increased circulating iron levels, elevated parenchymal iron stores and toxic iron species. Interestingly, recently developed hepcidin agonists prevented low hepcidin-induced toxicity, preclinically, thus demonstrating the potential of these compounds to prevent iron loading erythropoietic activity in MDS, especially in RS-MDS. ${ }^{25,40}$ Taken together, our data suggest a worsening over time of the ineffective erythropoiesis along with lower hepcidin levels in RS patients. ${ }^{41,42}$

Elevated NTBI levels could be demonstrated in our study early in the follow-up period of all patient groups. In iron loading anemias, such as thalassemia syndromes, it has been suggested that iron species, such as NTBI and LPI, serve as early indicators of iron toxicity and as measures for the effectiveness of iron chelation therapy in reducing potentially toxic iron molecules in the plasma. ${ }^{7,43}$ Excess toxic iron species catalyze the cellular generation of reactive oxygen species (ROS). Oxidative stress and high TSAT, as in combination with a subsequent decrease in cellular antioxidants, may lead to the oxidation of lipids, proteins and DNA, causing cell and tissue damage. ${ }^{44,45}$ Biomarkers of oxidative stress have been found to be increased in patients with MDS and iron overload. ${ }^{3,46-49}$ The combination of high serum ferritin levels as well as the presence of NTBI and LPI was noted to be more frequent in RS patients compared to non-RS patients in our study. Herein, it is important to realize that in general practice, including our study, serum samples are collected immediately prior to transfusions. LPI levels are usually elevated for a few days after transfusion (except when transferrin is highly saturated) in contrast to the more stable NTBI which have been reported to have a longer half-life..$^{50,51}$ These free iron molecules are easily translocated intracellularly and cause oxidative stress as shown in thalassemia. ${ }^{33}$ Oxidative stress may explain why elevated LPI levels are associated with an increased risk of dying prematurely; too early to die from causes related to classical iron overload in the lungs, liver and heart as observed in young thalassemia patients after long-term transfusions.

Less is known about the pathophysiology and tissue toxicity of iron overload caused by ineffective erythropoiesis in MDS. We observed that high NTBI and LPI levels also occurred in RS patients not receiving transfusions, indicating that iron toxicity (oxidative stress) may also occur in this category of MDS patients (Figure 4), similar to TI $\beta$-thalassemia intermedia, $\alpha$-thalassemia ( $\mathrm{Hb}-\mathrm{H}$ disease), and X-linked sideroblastic anemia. ${ }^{52,53}$

Previously, we reported that detectable LPI occurred almost exclusively in samples with TSAT $>80 \% .^{29}$ Interestingly, in the study herein, survival of patients with TSAT $>80 \%$ was not different from the survival of patients with a TSAT below this level (Online Supplementary Figure S1). The lowest hepcidin levels have been observed in RS patients, ${ }^{5}$ similar to our observations. The elevation of LPI in TI patients occurred exclusively in RS patients as expected in view of the low hepcidin levels leading to increased serum iron levels, through increased intestinal iron absorption and increased iron release from macrophages. Non-RS patients with SF3B1 mutations may show a similar iron pathophysiology since they appear to have a similar outcome compared to RS-MDS patients with SF3B1 mutations. ${ }^{54}$ In addition, significant relationships were found between SF3B1 mutations and marrow erythroblasts $(P=0.001)$ or soluble transferrin receptor factor 15 $(P=0.033) .{ }^{11}$ Our data show that elevated LPI levels - in contrast to elevated NTBI levels and TSAT - associate with decreased survival. The risk of dying prematurely in patients with detectable LPI levels occurred too early in this study to explain this risk by classical iron overload due to organ toxicity (lungs, liver and heart) after long term transfusions, but this indicates a direct effect associated with elevated LPI levels. The impact of detectable LPI was only significant in the large non-RS group, but the same tendency was observed in the smaller RS subpopulation. This effect was independent of ESA treatment, indicating that the effect of LPI on outcome is not simply an effect of the interaction of LPI with ESA, as a previously described outcome modifier. ${ }^{55,56}$ The widely used parameter TSAT cannot serve as a parameter to predict survival. However, TSAT can be used as a prescreening method to identify patients who are at risk to develop detectable LPI levels and associated poor prognosis. This approach may reduce the number of LPI determinations substantially.

Ferritin levels have been reported as a prognostic indicator in MDS, but ferritin as a marker of iron toxicity may be compromised by the stage of MDS, the cumulative transfusional load and its properties as an acute phase protein. ${ }^{57-59}$ Moreover, the level of ferritin does not indicate whether iron is stored in parenchymal cells or in the reticuloendothelial system (RES), of which the former is considered to be a more toxic form of iron overload. The foregoing is reflected by the weaker correlation of ferritin levels with LPI when compared with the correlation between TSAT and LPI levels. The positive correlation between CRP and hepcidin in the study here- 
in suggests that inflammation also influences iron homeostasis in some MDS patients, as reported for patients with other inflammatory diseases. ${ }^{4}$ Similar to ferritin, CRP had a significant impact on survival, potentially reflecting the impact of infections and autoimmune diseases on survival in this patient group. Finally, we demonstrated, in the limited number of patients treated with iron chelators in the study herein, that LPI levels decreased below LLOD, even in patients with high TSAT during treatment with deferasirox. These data corroborate with the post hoc data from a large chelation study in MDS. ${ }^{43}$

In conclusion, we demonstrated a disturbed iron homeostasis both in transfusion dependent MDS patients and in the subgroup of transfusion independent RS patients. This is the first clinical study that identifies LPI as a relevant marker for the potentially toxic fraction of iron species and its impact on OS. Increased LPI levels were restricted to patients with TSAT percentages exceeding $80 \%$. However, TSAT exceeding $80 \%$ alone was not prognostic for survival. Therefore, we propose TSAT as a screening parameter to assess risk for detectable LPI. Additional studies are warranted to show that intervention with iron chelation improves survival, comorbidities and quality of life in lower-risk MDS patients by lowering LPI levels.

\section{Acknowledgments}

The authors would like to thank the other members of the EUMDS Steering Committee: Pierre Fenaux, France; Moshe Mittelman, Israel; Reinhard Stauder, Austria; Guillermo Sanz, Spain; Luca Malcovati, Italy; Ulrich Germing, Germany; Krzysztof Madry, Poland; Mette Skov Holm, Denmark; Antonio Medina Almeida, Portugal; Aleksandar Savic, Republic of Serbia and Njetočka Gredelj Šimec, Croatia.

The authors and members of the steering committee of the EUMDS registry would like to thank all local investigators and operational team members for their contribution.

The authors wish to thank Erwin Wiegerinck of the Radboudumc expertise center for iron disorders for the measurement of LPI, NTBI and hepcidin-25, and Siem Klaver, Margot Rekers and Karin van der Linden for sample handling and Elise van Pinxten-van Orsouw and Linda van der Landen for data entry of all iron parameters.

\section{Funding}

The work of the EUMDS Registry for low and intermediate-1 MDS is supported by an educational grant from Novartis Pharmacy B.V. Europe. This work is part of the MDS-RIGHT activities, which has received funding from the European Union's Horizon 2020 research and innovation program under grant agreement No 634789 - "Providing the right care to the right patient with MyeloDysplastic Syndrome at the right time".

\section{References}

1. Bennett JM, Catovsky D, Daniel MT, et al. Proposals for the classification of the myelodysplastic syndromes. $\mathrm{Br} \mathrm{J}$ Haematol. 1982;51(2):189-199.

2. Greenberg P, Cox C, LeBeau MM, et al. International scoring system for evaluating prognosis in myelodysplastic syndromes. Blood. 1997;89(6):2079-2088.

3. de Swart L, Smith A, Johnston TW, et al. Validation of the revised international prognostic scoring system (IPSS-R) in patients with lower-risk myelodysplastic syndromes: a report from the prospective European LeukaemiaNet MDS (EUMDS) registry. Br J Haematol. 2015;170(3):372383.

4. Zipperer E, Post JG, Herkert M, et al. Serum hepcidin measured with an improved ELISA correlates with parameters of iron metabolism in patients with myelodysplastic syndrome. Ann Hematol. 2013; 92(12):1617-1623.

5. Santini V, Girelli D, Sanna A, et al. Hepcidin levels and their determinants in different types of myelodysplastic syndromes. PLoS One. 2011;6(8):e23109.

6. Cuijpers ML, Raymakers RA, Mackenzie MA, de Witte TJ, Swinkels DW. Recent advances in the understanding of iron overload in sideroblastic myelodysplastic syndrome. Br J Haematol. 2010;149(3):322333 .

7. Shenoy N, Vallumsetla N, Rachmilewitz E, Verma A, Ginzburg Y. Impact of iron overload and potential benefit from iron chelation in low-risk myelodysplastic syndrome. Blood. 2014;124(6):873-881.

8. Greenberg P, Cox C, LeBeau MM, et al. International scoring system for evaluating prognosis in myelodysplastic syndromes.
Blood. 1997;89(6):2079-2088.

9. Kautz L, Nemeth E. Molecular liaisons between erythropoiesis and iron metabolism. Blood. 2014;124(4):479-482

10. Ramirez JM, Schaad O, Durual S, et al Growth differentiation factor 15 production is necessary for normal erythroid dif ferentiation and is increased in refractory anaemia with ring-sideroblasts. $\mathrm{Br} J$ Haematol. 2009;144(2):251-262.

11. Ambaglio I, Malcovati L, Papaemmanuil E, et al. Inappropriately low hepcidin levels in patients with myelodysplastic syndrome carrying a somatic mutation of SF3B1. Haematologica. 2013;98(3):420-423.

12. Rund D, Rachmilewitz E. Beta-thalassemia. N Eng J Med. 2005;353(11):1135 1146.

13. Gattermann N, Rachmilewitz EA. Iron overload in MDS-pathophysiology, diagnosis, and complications. Ann Hematol. 2011;90(1):1-10.

14. Ganz T. Systemic iron homeostasis. Physiol Rev. 2013:93(4):1721-1741.

15. Cabantchik ZI. Labile iron in cells and body fluids: physiology, pathology, and pharmacology. Front Pharmacol. 2014:5:45.

16. Breuer W, Hershko C, Cabantchik ZI. The importance of non-transferrin bound iron in disorders of iron metabolism. TransfusSci. 2000;23(3):185-192.

17. Brissot P, Ropert M, Le Lan C, Loreal O. Non-transferrin bound iron: a key role in iron overload and iron toxicity. Biochim Biophys Acta. 2012;1820(3):403-410.

18. Hershko C, Graham G, Bates GW Rachmilewitz EA. Non-specific serum iron in thalassaemia: an abnormal serum iron fraction of potential toxicity. Br J Haematol. 1978;40(2):255-263

19. Ganz T. Hepcidin and iron regulation, 10 years later. Blood. 2011;117(17):4425-4433.

20. Girelli D, Nemeth E, Swinkels DW Hepcidin in the diagnosis of iron disorders.
Blood. 2016;127(23):2809-2813

21. Fleming RE, Ponka P. Iron overload in human disease. $\mathrm{N}$ Eng J Med. 2012;366(4):348-359.

22. Tanno T, Bhanu NV, Oneal PA, et al. High levels of GDF15 in thalassemia suppress expression of the iron regulatory protein hepcidin. Nat Med. 2007;13(9):1096-1101.

23. Porter JB, de Witte $T$, Cappellini MD Gattermann N. New insights into transfusion-related iron toxicity: Implications for the oncologist. Crit Rev Oncol Hematol. 2016;99:261-271.

24. Nemeth E. Hepcidin and beta-thalassemia major. Blood. 2013;122(1):3-4.

25. Metzgeroth G, Rosee PL, Kuhn C, et al. The soluble transferrin receptor in dysplastic erythropoiesis in myelodysplastic syndrome. Eur J Haematol. 2007;79(1):8-16.

26. Speeckaert MM, Speeckaert R, Delanghe JR. Biological and clinical aspects of soluble transferrin receptor. Crit Rev Clin Lab Sci. 2010;47(5-6):213-228.

27. Huebers HA, Beguin $Y$, Pootrakul $P$ Einspahr D, Finch CA. Intact transferrin receptors in human plasma and their relation to erythropoiesis. Blood. 1990; 75(1):102-107

28. Khatami S, Dehnabeh SR, Mostafavi E, et al. Evaluation and comparison of soluble transferrin receptor in thalassemia carriers and iron deficient patients. Hemoglobin. 2013;37(4):387-395.

29. de Swart L, Hendriks JC, van der Vorm LN, et al. Second international round robin for the quantification of serum non-transferrin-bound iron and labile plasma iron in patients with iron-overload disorders. Haematologica. 2016;101(1):38-45.

30. Evans RW, Rafique R, Zarea A, et al. Nature of non-transferrin-bound iron: studies on iron citrate complexes and thalassemic sera. J Biol Inorg Chem. 2008;13(1):57-74.

31. Silva AM, Hider RC. Influence of non- 
